\title{
NEUTRALINO CLUMPS AND COSMIC RAYS
}

\author{
P. SALATI* \\ LAPTH, 9 Chemin de Bellevue, BP110, \\ F-74941 Annecy-le-Vieux Cedex, France \\ *E-mail: salati@lapp.in2p3.fr \\ http://lappweb.in2p3.fr/ salati/ \\ Lapth-Conf-1172/07 \\ Proceedings of the 6th International Workshop on the Identification of Dark Matter, \\ held in Rhodes Island, Greece, September 11-16, 2006
}

\begin{abstract}
The halo of the Miky Way might contain numerous and dense substructures inside which the putative weakly interacting massive particles (suggested as the main constituent of the astronomical dark matter) would produce a stronger annihilation signal than in the smooth regions. The closer the nearest clump, the larger the positron and antiproton cosmic ray fluxes at the Earth. But the actual distribution of these substructures is not known. The predictions on the antimatter yields at the Earth are therefore affected by a kind of cosmic variance whose analysis is the subject of this contribution. The statistical tools to achieve that goal are presented and Monte Carlo simulations are compared to analytic results.

Keywords: Dark Matter Clumps; Neutralinos; Antimatter Cosmic Rays
\end{abstract}

\section{Motivations}

The universe contains a significant fraction of invisible and non-baryonic matter ${ }^{1}$ whose nature is still unresolved. This dark matter could be made of weakly interacting massive particles, ${ }^{2}$ such as the supersymmetric neutralinos, whose annihilations inside the galactic halo might produce a sizeable yield of positrons and antiprotons. These rare antimatter cosmic rays will be detected with improved accuracy by the forthcoming experiments. ${ }^{3,4} \mathrm{Nu}-$ merical simulations of structure formation indicate that dark matter (DM) is clumpy on small scales. ${ }^{5}$ Because neutralinos are more tightly packed inside the DM substructures, their mutual annihilation is enhanced. The resulting increase of the associated antimatter signals at the Earth has been so far accounted for by an overall boost factor and by shifting upwards the 
J.Lavalle, J.Pochon, P.Salati \& R.Taillet (2006)

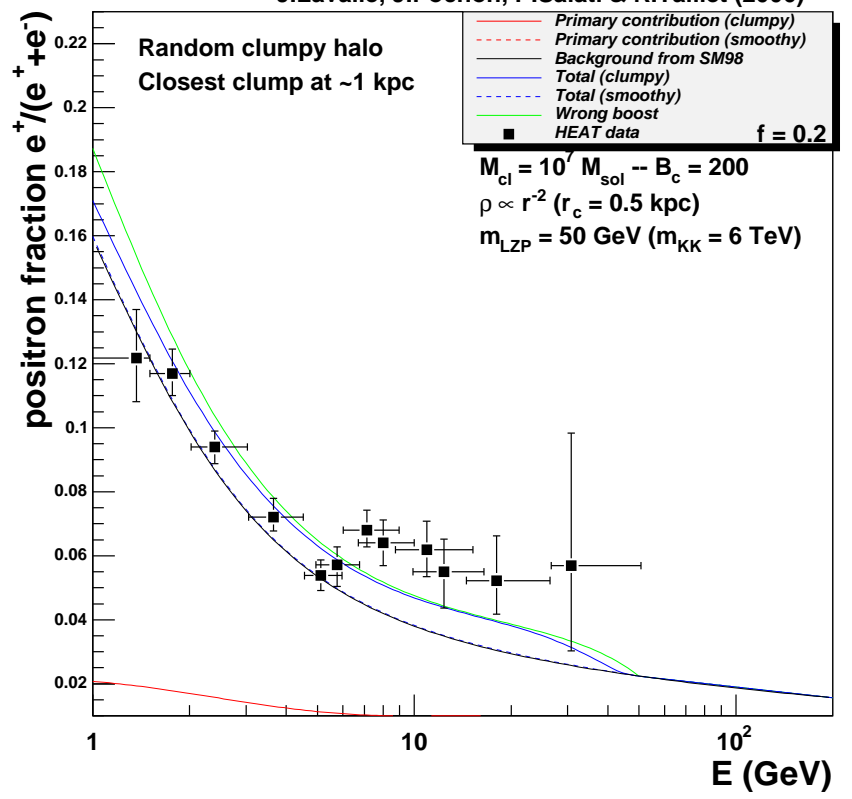

J.Lavalle, J.Pochon, P.Salati \& R.Taillet (2006)

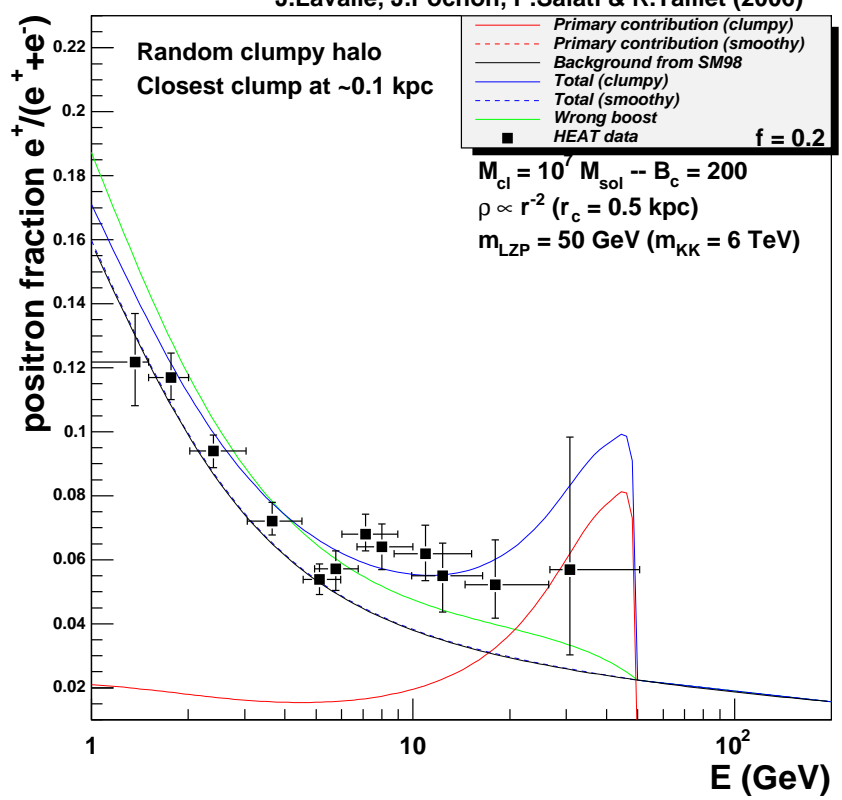

Fig. 1. The positron fraction is derived for a $50 \mathrm{GeV}$ Kaluza-Klein inspired particle ${ }^{6}$ and compared to the HEAT excess ${ }^{7}$ which cannot be explained by the pure conventional background ${ }^{8}$ in black. An isothermal profile has been assumed for the dark matter halo of the Milky Way, a fraction $f=0.2$ of which lies in clumps with mass $10^{7} \mathrm{M}_{\odot}$ and intrinsic boost $B_{c}=200$. Two random realizations of that clumpy DM halo are featured in blue. The distance of the closest substructure has been set equal to $1 \mathrm{kpc}$ in the left panel and decreased to $0.1 \mathrm{kpc}$ in the right panel. The green curve corresponds to the traditional and wrong shift by a factor of $f \times B_{c}=40$ of the positron spectrum which a completely smooth DM halo would yield. 
cosmic ray fluxes obtained in the case of a smooth distribution of DM particles.

This approach is too simple. If a substructure were to lie in our immediate vicinity, we would observe a significantly distorted spectrum as illustrated in Fig. 1 for a $50 \mathrm{GeV}$ LZP species. The question then naturally arises to know if such a possibility is probable or very rare. Should we know the exact location of each DM clump, the positron and antiproton spectra would be derived exactly. On the contrary, they are affected by a kind of cosmic variance because an infinite number of different halo realizations are possible. The theoretical predictions on the detectable fluxes at the Earth must take into account that lack of knowledge.

We have therefore built specific tools ${ }^{9}$ to address this issue. We will concentrate here on the pedagogical example where a positron line is produced through the mutual annihilations of the DM particles $\chi$ into $e^{+} e^{-}$pairs. This scenario is plausible in the framework of Kaluza-Klein inspired theories. In the absence of any substructure, the DM distribution $\rho_{s}$ is smooth and the resulting flux is given by

$$
\phi_{s}(E)=\mathcal{S} \int_{\text {DM halo }} G(\mathbf{x})\left\{\frac{\rho_{s}(\mathbf{x})}{\rho_{0}}\right\}^{2} d^{3} \mathbf{x}
$$

where the factor $\mathcal{S}$ is defined as

$$
\mathcal{S}=\frac{1}{8 \pi} v_{e^{+}}(E)\left\langle\sigma_{\text {ann }}\left(\chi \chi \rightarrow e^{+} e^{-}\right) v\right\rangle\left(\frac{\rho_{0}}{m_{\chi}}\right)^{2} .
$$

The probability for a positron injected at $\mathbf{x}$ to propagate towards the Earth which it reaches with the degraded energy $E \leq E_{S}$ is described by the Green function $G(\mathbf{x})$. In the presence of clumps, the DM distribution is given by the superposition $\rho=\rho_{s}^{\prime}+\delta \rho$ where a smooth component $\rho_{s}^{\prime}$ is still present while $\delta \rho$ accounts for the substructures. The propagator $G$ has already been discussed in the literature. ${ }^{10}$ Positrons loose energy through synchrotron radiation and inverse Compton scattering on stellar light and on the CMB. The farther they originate, the smaller their energy $E$ at the Earth for a fixed value of $E_{S}$. Positrons that are detected at the energy $E$ have been produced within a typical range $\lambda_{\mathrm{D}}$ which decreases as $E$ increases towards $E_{S}$ but is nevertheless much larger than the size of the DM substructures. The positron flux becomes

$$
\phi(E)=\phi_{s}^{\prime}+\left(\phi_{r}=\sum_{i} \varphi_{i}\right)
$$


where the contribution from the ith clump is $\varphi_{i}=\mathcal{S} \times G\left(\mathbf{x}_{i}\right) \times \xi_{i}$. That minihalo produces as many positrons as if the entire volume

$$
\xi_{i} \equiv \frac{B_{i} M_{i}}{\rho_{0}}=\int_{\text {ith clump }}\left\{\frac{\delta \rho(\mathbf{x})}{\rho_{0}}\right\}^{2} d^{3} \mathbf{x}
$$

were filled with the density $\rho_{0}$. The volume $\xi_{i}$ can also be expressed as a function of the substructure mass $M_{i}$ and intrinsic boost $B_{i}$.

An infinite set of halo realizations must be taken into account, each of which produces a different flux $\phi_{r}$. The boost factor $B=\phi / \phi_{s}$ at the Earth is not unique and must be treated as a random variable. We present in the next section a procedure to determine the statistical law according to which $B$ is distributed and will show that its average value and variance depend on the energy $E$.

\section{Computing the Odds of the Galactic Lottery}

The statistical properties of the random flux $\phi_{r}$ and of the associated boost factor $B$ have been thoroughly investigated in a recent analysis. ${ }^{9}$ I will just point out its salient features and summarize the hypotheses on which it is based.

(i) To simplify the discussion and without loss of generality, we assume identical clumps with mass $M_{c}$ and intrinsic boost $B_{c}$. The random flux $\phi_{r}$ simplifies into

$$
\phi_{r}=\mathcal{S} \times \xi_{c} \times \sum_{i} G_{i},
$$

where the effective volume $\xi_{c}=B_{c} M_{c} / \rho_{0}$ is the same for each minihalo.

(ii) The actual distribution of DM substructures is one particular realization to be taken from the statistical ensemble made up by all the possible random distributions. The flux contributed by the clumps and the boost factor must be averaged on that infinite set to yield $\left\langle\phi_{r}\right\rangle$ and $B_{\text {eff }}=\left\langle B=\left(\phi / \phi_{s}\right)\right\rangle$ whereas the variance is defined as

$$
\sigma_{r}^{2}=\left\langle\phi_{r}^{2}\right\rangle-\left\langle\phi_{r}\right\rangle^{2} \quad \text { and } \quad \sigma_{B}=\sigma_{r} / \phi_{s} .
$$

(iii) Clumps are distributed independently of each other. This is a strong assumption that generally holds because the substructure correlation length is smaller than the propagation range $\lambda_{\mathrm{D}}$. We just need then to determine how a single clump is distributed inside the Milky Way halo in order to derive the statistical properties of an entire population of $N_{H}$ such substructures. If $\varphi$ denotes the contribution of a single minihalo, we get

$$
\left\langle\phi_{r}\right\rangle=N_{H}\langle\varphi\rangle \text { and } \sigma_{r}^{2}=N_{H} \sigma^{2}=N_{H}\left\{\left\langle\varphi^{2}\right\rangle-\langle\varphi\rangle^{2}\right\} .
$$




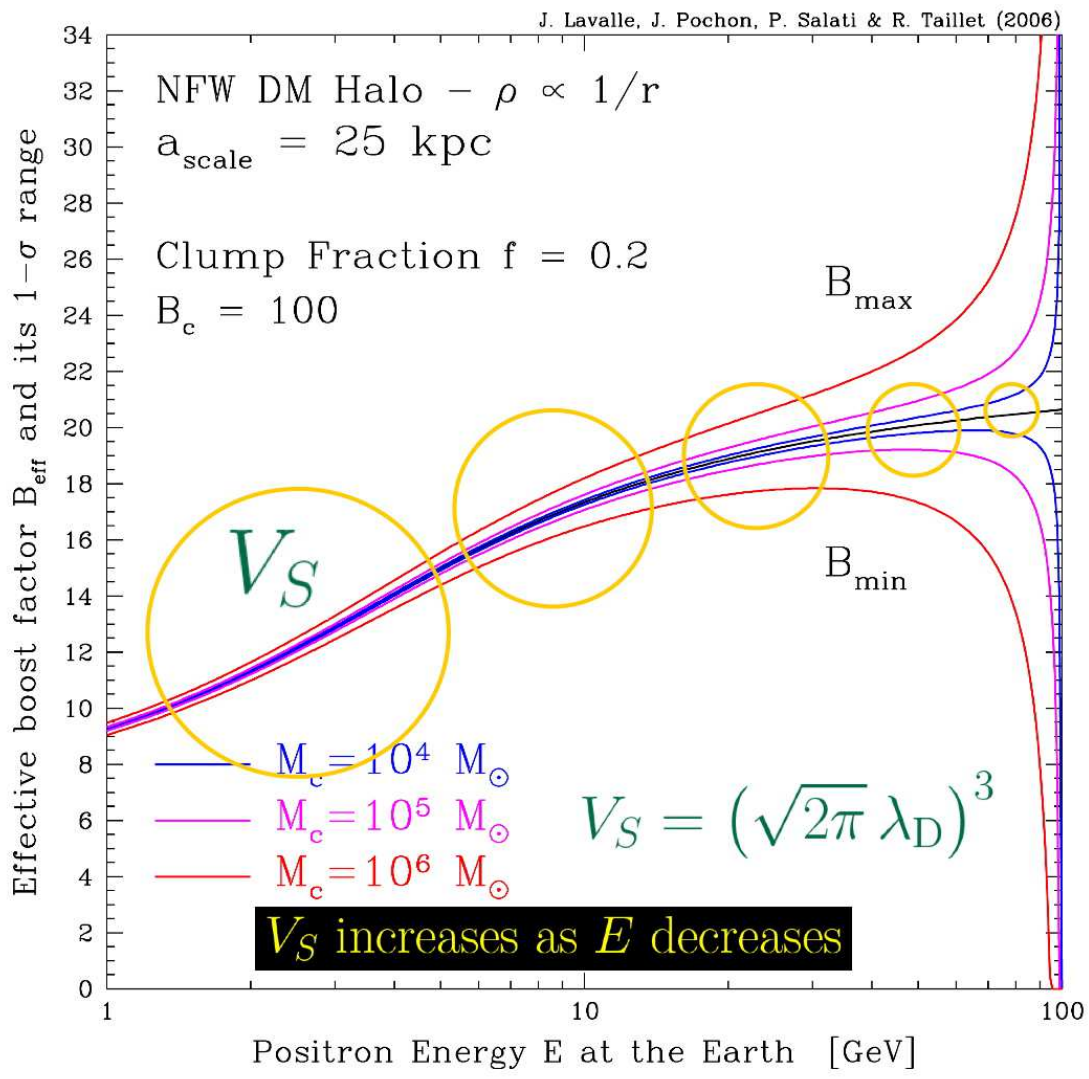

Fig. 2. Positrons are injected with the energy $E_{S}=100 \mathrm{GeV}$ and detected at the energy $E$. The effective boost factor $B_{\text {eff }}$ (black line) accounts for the average enhancement of the positron signal resulting from DM clumpiness. Although it has been so far considered to behave as a constant, the boost actually depends on the energy. Furthermore, as $E$ approaches $E_{S}$, the boost variance $\sigma_{B}$ increases significantly. The region from which the positrons detected at the Earth originate shrinks and the number $N_{S}$ of clumps which it contains decreases.

(iv) The set of the random distributions of one single clump inside the Milky Way halo $\mathcal{D}_{H}$ makes up the statistical ensemble $\mathcal{T}$ which we eventually need to consider. An event from that ensemble consists in a clump located at position $\mathbf{x}$ within the elementary volume $d^{3} \mathbf{x}$ and is weighted by the probability $p(\mathbf{x}) d^{3} \mathbf{x}$. The spatial distribution $p(\mathbf{x})$ and the flux density 
of probability $\mathcal{P}(\varphi)$ are related by

$$
\mathcal{P}(\varphi) d \varphi=d P=\int_{\mathcal{D}_{\varphi}} p(\mathbf{x}) d^{3} \mathbf{x}
$$

where $\mathcal{D}_{\varphi}$ is the region of the halo in which a clump must lie in order to contribute a flux $\varphi$ at the Earth. We readily conclude that any function $\mathcal{F}$ of the flux $\varphi$ (for instance $\varphi$ itself or its square $\varphi^{2}$ ) is given on average by

$$
\langle\mathcal{F}\rangle=\int \mathcal{F}(\varphi) \mathcal{P}(\varphi) d \varphi=\int_{\mathcal{D}_{H}} \mathcal{F}\{\varphi(\mathbf{x})\} p(\mathbf{x}) d^{3} \mathbf{x}
$$

Once $p(\mathbf{x})$ is known, $B_{\text {eff }}$ and $\sigma_{B}$ can be derived.

\section{Results and Perspectives}

We have applied that procedure in the case where $\rho_{s}^{\prime} \equiv(1-f) \times \rho_{s}$ and have finally assumed that the clumps trace the smooth DM distribution (which is not the case in general) by imposing that $p(\mathbf{x}) \propto \rho_{s}(\mathbf{x})$. The main results are summarized in Fig. 2 and 3.

To commence, the average boost $B_{\text {eff }}$ depends on the energy. As $E$ decreases, the part of the halo which contributes effectively to the positron signal at the Earth grows. Regions located close to the galactic center come into play, with a much larger density $\rho_{s}$ than in the solar neighborhood. At fixed intrinsic boost $B_{c}$, neutralino annihilation inside clumps is relatively less enhanced in these dense regions than in our vicinity, hence the behaviour exhibited in Fig. 2.

We also find that the boost variance $\sigma_{B}$ increases significantly as $E$ approaches $E_{S}$. The above mentioned domain of the halo, inside which the positrons detected at the Earth with the energy $E$ have been produced, has a typical volume $V_{S}=\left(\sqrt{2 \pi} \lambda_{\mathrm{D}}\right)^{3}$ which shrinks as $E$ gets near to $E_{S}$. The number $N_{S}$ of the substructures which it contains falls down and the boost variance increases because

$$
\frac{\sigma_{r}}{\left\langle\phi_{r}\right\rangle} \simeq \frac{\sigma_{B}}{B_{\text {eff }}} \simeq \frac{1}{\sqrt{N_{S}}} .
$$

Finally, in the large $N_{S}$ regime, the boost distribution follows a Maxwellian law as is featured in Fig. 3. This result may be derived with the help of the central limit theorem. In the opposite regime where $N_{S} \leq 1$, the boost distribution is driven by the product $N_{H} \times \mathcal{P}(\varphi)$ where the density of probability $\mathcal{P}(\varphi)$ for the flux generated by a single clump comes into play.

The method outlined here can be applied in particular to the intermediate mass black hole scenario. ${ }^{11}$ The spatial distribution $p(\mathbf{x})$ no longer 
traces the smooth density $\rho_{s}$. An effective boost $B_{\text {eff }}$ of $\sim 2$ to $3 \times 10^{3}$ is found ${ }^{12}$ for both antiprotons and positrons with a large variance.

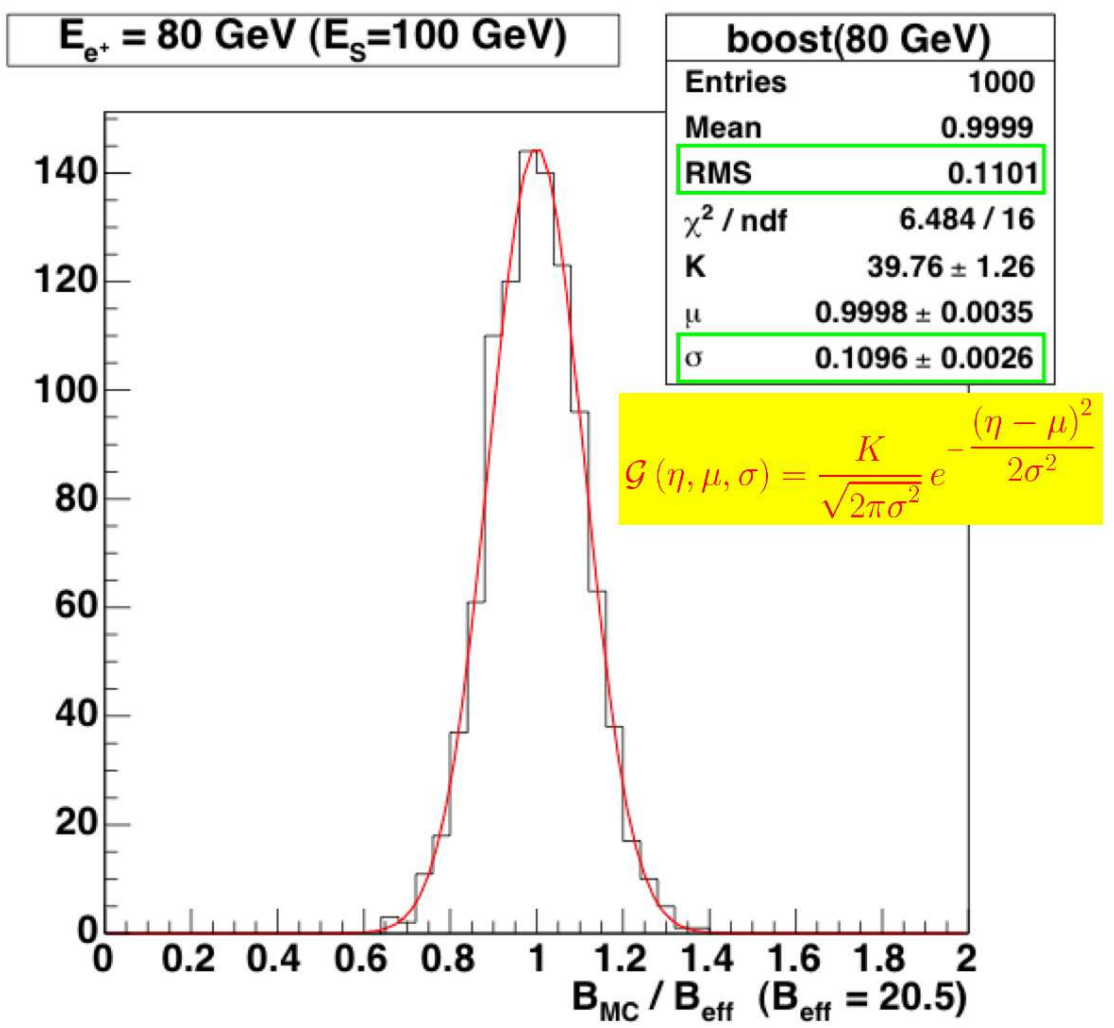

Fig. 3. In this Monte-Carlo simulation, clumps contribute a fraction $f=0.2$ to the mass of the Milky Way DM halo (assumed to follow a NFW profile with a scale radius of $25 \mathrm{kpc}$ ). Each substructure has a mass of $10^{5} \mathrm{M}_{\odot}$. A thousand different realizations of the DM halo have been generated here, each of them involving 271,488 clumps. The positron injection energy is $E_{S}=100 \mathrm{GeV}$. The number of realizations has been plotted as a function of the boost ratio $\eta=B / B_{\text {eff }}$. The rms value of 0.1101 yielded by the MonteCarlo for $\eta$ is in excellent agreement with the anaytic value $\sigma_{\eta}=\sigma_{B} / B_{\text {eff }}=0.1097$.

\section{Acknowledgments}

I would like to thank the organizers for the warm and friendly atmosphere of this exciting and inspiring conference as well as for their financial support. 
This work could not have been performed without the help of the french programme national de cosmologie PNC.

\section{References}

1. D. N. Spergel et al., astro-ph/0603449 (2006).

2. G. Bertone, D. Hooper and J. Silk, Phys. Rept. 405, 279 (2005).

3. F. Barao [AMS-02 Collaboration], Nucl. Instrum. Meth. A 535, 134 (2004).

4. P. Picozza et al., astro-ph/0608697 (2006).

5. J. Diemand, B. Moore and J. Stadel, Nature 433, 389 (2005).

6. K. Agashe and G. Servant, Phys. Rev. Letters 93, 231805 (2004).

7. S. Coutu et al., Positron Measurements with the Heat-Pbar Instrument, in International Cosmic Ray Conference, 2001.

8. I. V. Moskalenko and A. W. Strong, Astrophys. J. 493, 694 (1998).

9. J. Lavalle, J. Pochon, P. Salati and R. Taillet, astro-ph/0603796, to be published in A\&A (2007).

10. E. A. Baltz and J. Edsjo, Phys. Rev. D59, p. 023511 (1999).

11. G. Bertone, A. R. Zentner and J. Silk, Phys. Rev. D72, p. 103517 (2005).

12. P. Brun, G. Bertone, J. Lavalle, P. Salati and R. Taillet, in preparation (2007). 